\title{
Perseveration in Extinction and in Discrimination Reversal Tasks Following Selective Frontal Ablations in Macaca Mulatta
}

\author{
CHARLES M. BUTTER \\ Psychological Laboratories, University of Michigan, Amn Arbor, U.S.A.
}

(Received 8 October 1968)

\begin{abstract}
BUTTER, C. M. Perseveration in extinction and in discrimination reversal tasks following selective frontal ablations in Macaca mulatta. Physiol. Behav. 4 (2) 163-171, 1969.-In order to determine whether the perseverative syndrome exhibited by monkeys with frontal lobe lesions can be fractionated by partial frontal lesions, monkeys with dorsolateral frontal lesions, those with orbital frontal lesions, and those with partial orbital lesions were tested in three tasks: extinction of a food rewarded bar pressing response, spatial discrimination reversals and object discrimination reversals. Perseveration of barpressing in extinction was selectively related to the posteromedial or "limbic" portion of orbital frontal cortex. Deficits in object discrimination reversal, on the other hand, were specifically associated with the lateral sector of orbital cortex and possibly to the adjoining ventral portion of dorsolateral frontal cortex as well. Impairment in spatial discrimination reversal was most severe in monkeys with dorsolateral frontal removal. These findings suggest that the perseverative effects of orbital frontal ablation can be fractionated into at least two components and further support the view that dorsolateral frontal cortex is associated with spatial factors.
\end{abstract}

$\begin{array}{lccc}\text { Dorsolateral frontal cortex } & \text { Orbital frontal cortex } \\ \text { Object discrimination reversal } & \text { Rhesus monkey }\end{array}$ Extinction Spatial discrimination reversal

ReMOVAL of prefrontal cortex in monkeys produces perseverative tendencies in a variety of problem-solving and instrumental situations [10, 14]. Moreover, there is considerable evidence that the orbital surface of the frontal lobes provides the focus of these impairments. Thus, compared to monkeys with dorsolateral frontal (DLF) lesions, monkeys with orbital frontal (OF) lesions have difficulty in suppressing strong response tendencies in differentiation learning [2], learning set, and discrimination reversal tasks [10]. Moreover, OF monkeys, unlike those with DLF lesions, are impaired in extinction of a food-rewarded instrumental response [4].

The present study is addressed to the problem of further specifying the neural focus of these impairments and determining whether perseverative tendencies exhibited in different tasks are selectively related to particular frontal cortical regions. Several lines of evidence suggest that the posteromedial sector of OF cortex, referred to as area 13 by Walker [15] and as area FF by von Bonin and Bailey [1], may be the focus of at least some of the impairments in suppressing responses produced by total OF removal. For, both anatomical [11] and physiological [8] investigations indicate that this region is selectively related to the amygdala, removal of which produces impairments in extinction [16] and in discrimination reversal performance $[5,9]$ like those produced by total OF removal. Thus, in the present experi- ments, monkeys with selective ablation of posteromedial orbital cortex (PMOF) were compared to those with selective ablation of other OF sectors, as well as with monkeys with total OF and those with total DLF ablation, in extinction of a food-rewarded response and in object discrimination reversal. In addition, these animals were also tested in spatial discrimination reversals in an attempt to confirm the view that DLF cortex ablation is selectively associated with deficits in spatial tasks [10].

\section{EXPERIMENT I}

METHOD

\section{Subjects}

Subjects were 25 experimentally naive rhesus monkeys (Macaca mulatia) of both sexes, ranging in weight from $4.1-6.7 \mathrm{~kg}$. Because the lesions in 5 operated animals extended considerably beyond the intended boundaries, only the data for the remaining 20 animals are presented. Throughout the three experiments monkeys were housed in indvidual cages and maintained on a diet of Purina Monkey Chow ( $45 \mathrm{cal} / \mathrm{kg}$ day) supplemented with fresh fruit on weekends.

\footnotetext{
${ }^{1}$ This research was supported by a grant (GB-1603) from the National Science Foundation. The author acknowledges the assistance of Neal Barmack and William Chilton who tested the animals and Dianne Allmen who was responsible for the histology.
} 


\section{Apparatus}

Animais were trained in an operant chamber measuring $52 \mathrm{~cm}$ on each side and illuminated with a $25 \mathrm{~W}$ overhead light. A metal telegraph key, $5 \mathrm{~cm}$ long, projected into the chamber $15 \mathrm{~cm}$ above the floor. An automatic pellet dispenser, located outside the chamber, was connected by a spring tube to a plastic food tray located $7 \mathrm{~cm}$ below the lever. Each time a food pellet was delivered, either by experimenter's manual switch or by the animal depressing the lever, the tray was illuminated with a $0.5 \mathrm{~W}$ bulb and the overhead light was turned off for $2.5 \mathrm{sec}$. Food pellets were whole diet composition (D and $G$ Monkey Pellets, each weighing $0.75 \mathrm{~g}$ ). The operant chamber was located in a sound-attenuated enclosure, and outside noises were masked by white noise delivered to an overhead speaker. Lever presses were recorded on digital counters and a cumulative recorder, and the delivery of food pellets was automatically programmed with timers and relays.

\section{Procedure}

Preoperative training. Following a two-day fast, the animals were trained to lever press in order to obtain food pellets. Lever-press training required from one to four daily sessions, and on the last session monkeys were allowed to obtain $30-40$ pellets on a continuous reinforcement schedule.

Following lever-press training, the animals were trained in 30 min daily sessions to lever press on a VI-1 min reinforcement schedule. In the first three sessions, each reinforcement was locked in for an unlimited period of time during which a lever press produced delivery of a pellet. However, on all subsequent sessions, a limited hold was placed on the VI schedule, so that each reinforcement was obtainable only during a particular time interval. On each session this time interval was equivalent to the monkey's mean inter-response interval on the prior session. This procedure tended to selectively reinforce higher and more stable rates of responding on successive sessions. Daily VI training sessions continued until the animals' responses on three days were between 25 and 35 per cent of their responses on the last 10 days. The animals were then assigned to six groups matched for response rates on the last three days of VI training: an unoperated group $(N)(N=4)$, a dorsolateral frontal group (DFL) $(N=4)$, an orbital frontal group (OF) $(N=3)$, a posteromedial orbital frontal group (PMOF) $(\mathrm{N}=3)$, a lateral orbital frontal group (LOF) $(\mathrm{N}=3)$, and an anterior orbital frontal group (AOF) $(\mathrm{N}=3)$.

Surgery. Surgery was carried out under aseptic conditions following intraperitoneal administration of sodium pentobarbital $(45 \mathrm{mg} \mathrm{kg}$ ). Cortical removals, which were all bilateral. were made through openings rongeured in the frontal bone; for several of the orbital frontal lesions, a large oval shaped portion of the anterior calvarium was cut out and later replaced and secured to the intact bone with wire. Cortical resections were performed with an 18-gauge sucker, and bleeding was controlled with cottonoid and thermocoagulation.

The DLF removals were intended to include all cortex on the dorsolateral surface from the anterior banks of the arcuate sulcus to the frontal pole, including the depths of sulcus principalis. The dorsal boundary of the DLF lesion was the longitudinal fissure and the convexity bordering the ventral surface was the ventral boundary. The OF removals were intended to include all cortex on the ventral surface of the frontal lobes, including the depths of the orbital sulci and extending medially to inciude gyrus rectus and laterally to the convexity bordering the dorsolateral surface. The PMOF lesions were intended to selectively remove the medial posterior region referred to as area FF by von Bonin and Bailey [1]. The LOF lesion was intended to remove the posterolateral portion of orbital frontal cortex, extending medially to the lateral limit of the PMOF region and to a line extending anteriorly from this border. The AOF lesion was intended to remove all orbital cortex anterior to the PMOF lesions and medial to the LOF lesions. (See Fig. 1. for the intended boundaries of the partial orbital lesions.) Wounds were closed in anatomical layers with silk sutures, and bicillin (500,000 units) was administered following surgery.

Postoperative testing. Ten days to two weeks following surgery, and following a comparable rest period for the $\mathrm{N}$ monkeys, the animals were again administered daily sessions of VI reinforcement as they were preoperatively, but now a $1 \mathrm{hr}$ extinction period immediately followed each $30 \mathrm{~min}$ VI reinforcement session. Daily sessions of VI reinforcement followed by extinction were continued for 10 days.
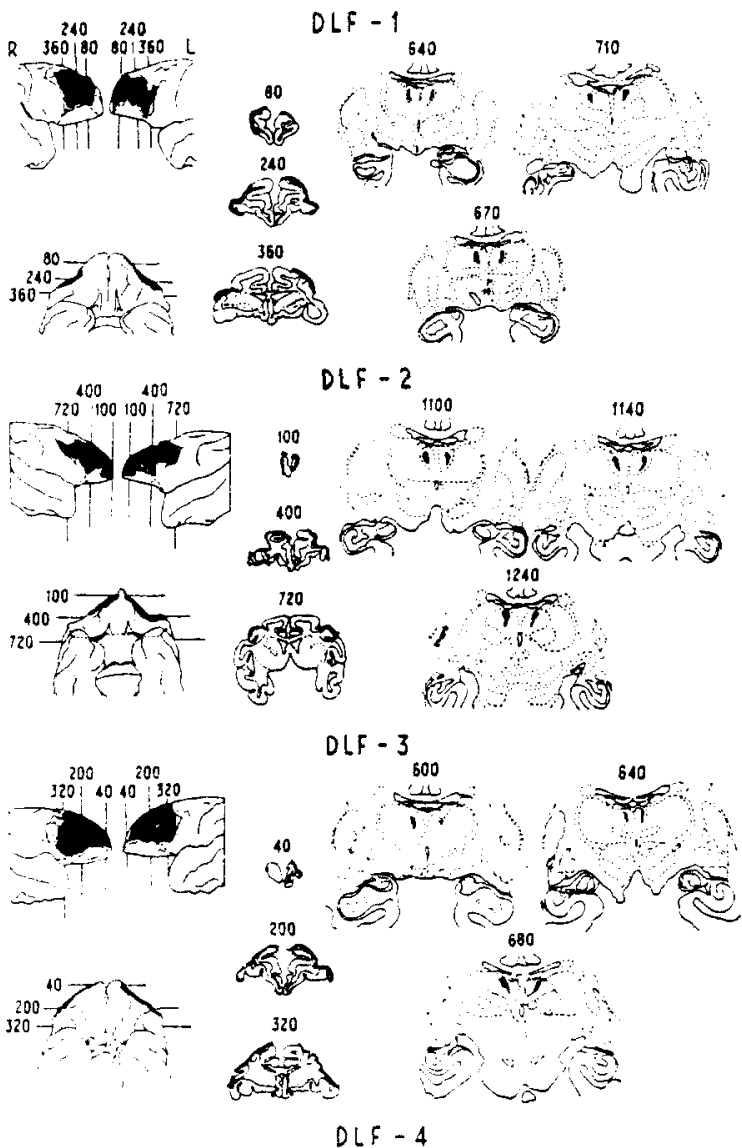

580
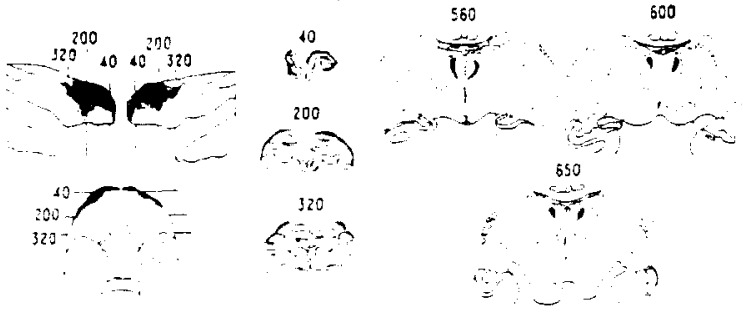

FIG. $1 \mathrm{~A}$ 
OF -1
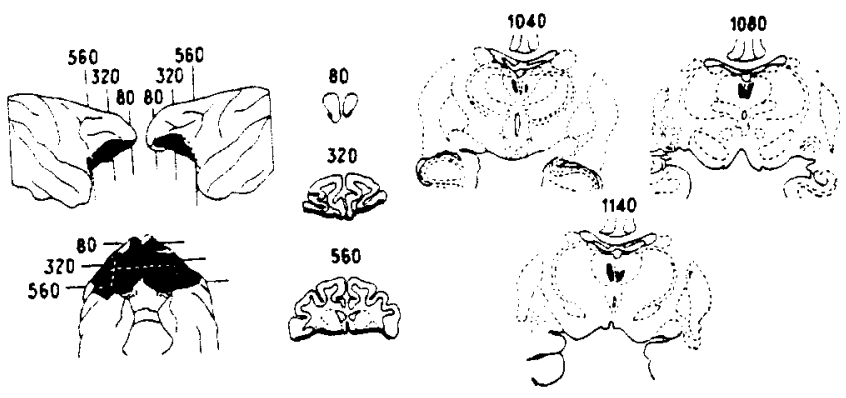

$O F-2$
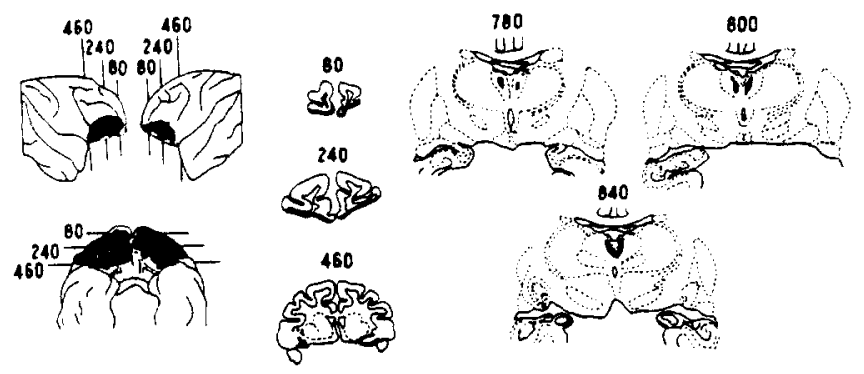

OF -3
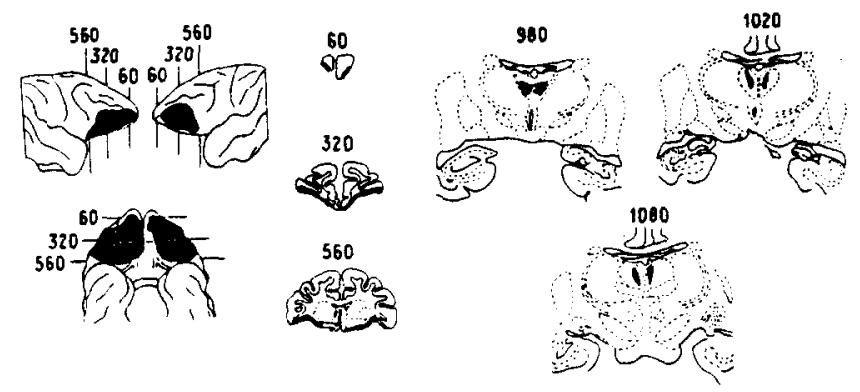

FIG. 1B

Histology. Following the completion of testing in Experiment 3 , the operated animals were deeply anesthetized with sodium pentobarbital and then perfused intracardially with $0.9 \%$ saline followed by $10 \%$ formalin. Their brains were then removed and prepared for embedding in celloidin. Transverse sections $40 \mu$ thick were cut, and every tenth section was stained with thionine.

Figure 1 shows reconstructions of the lesions from ventral and lateral views, along with representative cross sections through the lesions and through portions of the thalamus showing retrograde degeneration. It will be noted that the reconstructions from the ventral view of the complete and partial OF ablations show the intended boundaries of the AOF, LOF and PMOF regions in dashed lines. Also, all the reconstructions from the lateral view show in dashed lines the position of the lip separating the dorsolateral from the ventral surface. These boundaries were drawn on the reconstructions before the lesions were plotted, so that the shape and extent of the lesions did not influence the setting of the boundaries.
The DLF lesions included virtually all cortex anterior to the arcuate sulcus including the depths of sulcus principalis, and they only minimally involved the orbital surface. These lesions produced retrograde degeneration confined to the lateral portion of $n$. medialis dorsalis (see Fig. 1A). The OF removals were virtually complete and showed only slight involvement of the dorsolateral surface. Likewise, there was slight and variable invasion of the olfactory tubercle and of the ventral portion of the putamen and caudate nucleus in these brains. The OF lesions produced retrograde degeneration in the medial division of $n$. medialis dorsalis (see Fig. 1B). Figures 1C-E show that each of the partial lesions involved extensive or complete removal of the intended region without extensive damage to neighboring orbital regions. On the other hand, all the LOF lesions produced damage to the dorsolateral cortex, and in LOF-1 this damage was extensive (see Fig. 1C). Moreover, the PMOF lesions, like the total OF lesions, involved slight damage to nearby subcortical structures and to the olfactory tubercle (see Fig. 1D). With regard to retrograde degeneration following the partial OF removals, 


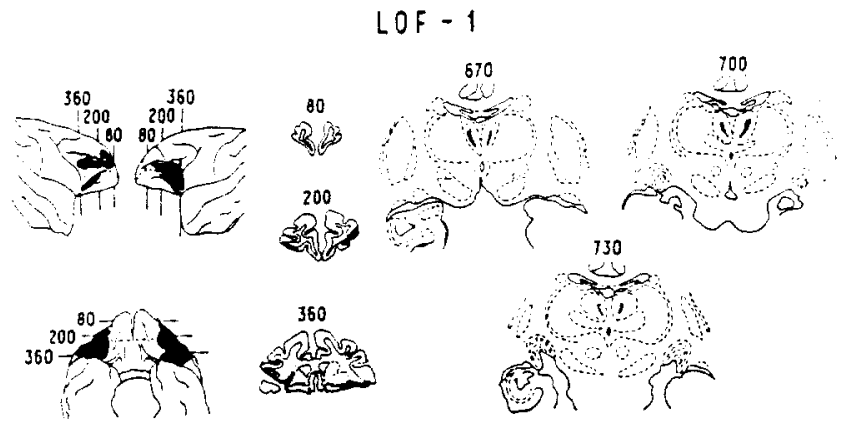

LOF -2
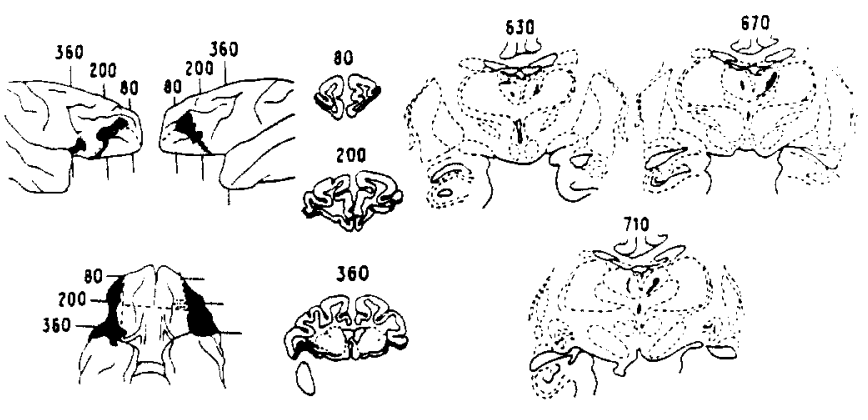

LOF -3
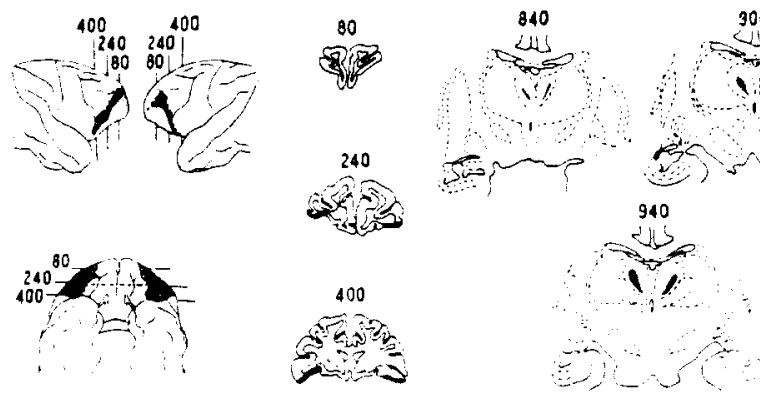

FIG. 1C

only the PMOF removals produced cell loss in the medial division of $n$. medialis dorsalis, confirming the observations of Pribram, Chow and Semmes [12]. The LOF lesions produced retrograde degeneration confined to the lateral division of $n$. medialis dorsalis, although there was less involvement of the dorsal portion of this nucleus than there was in the DLF brains (see Fig. 1C). This degeneration is apparently due to LOF damage as well as to DLF damage, for the lesion on the left side of LOF-3, which involved only slight damage to DLF cortex, produced cell loss similar to that seen in the other LOF brains involving more extensive DLF damage. Finally, as seen in Fig. IE, the AOF lesions produced only slight and variable regions of degeneration largely in the dorsal portion of $n$. medialis dorsalis.

\section{RESULTS AND DISCUSSION}

The response rates of the six groups in the last three preoperative and in the 10 postoperative VI-reinforcement sessions are shown in Fig. 2. The groups, which were fairly well matched for response rates preoperatively, all showed response decrements following surgery and following the two-week rest period for the $\mathrm{N}$ group. However, over the course of the 10 postoperative sessions, all groups increased their response rates and reattained preoperative levels of responding. Although the mean scores of the operated groups, notably the LOF and PMOF groups, deviated from those of the $\mathbf{N}$ group, there was considerable overlap of individual animals" scores in all groups. Consequently, no significant group effect was found in an analysis of variance of these data.

On the other hand, significant group differences were found in extinction (see Fig. 3). Both the OF and PMOF groups increased their responses over the first 6 sessions, whereas the remaining operated groups, like the $\mathrm{N}$ group, failed to show noticeable trends in extinction responses over the 10 sessions. These group differences were revealed in an analysis of variance as a significant groups effect $(F=3.86$ : $d f=5: 14 ; p<0.05)$ and groups by sessions interaction $(\mathrm{F}=$ 


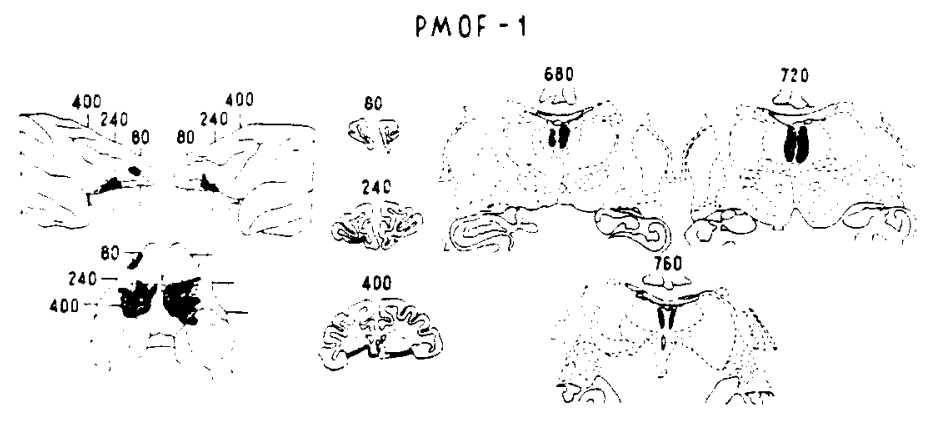

PMOF - 2
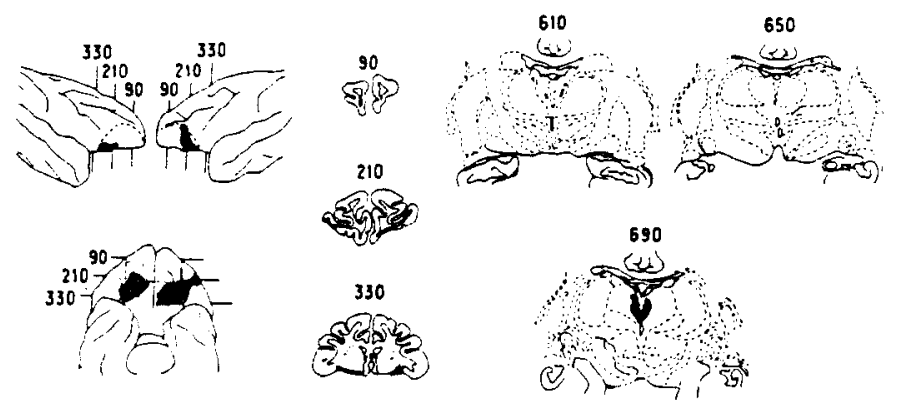

PMOF - 3
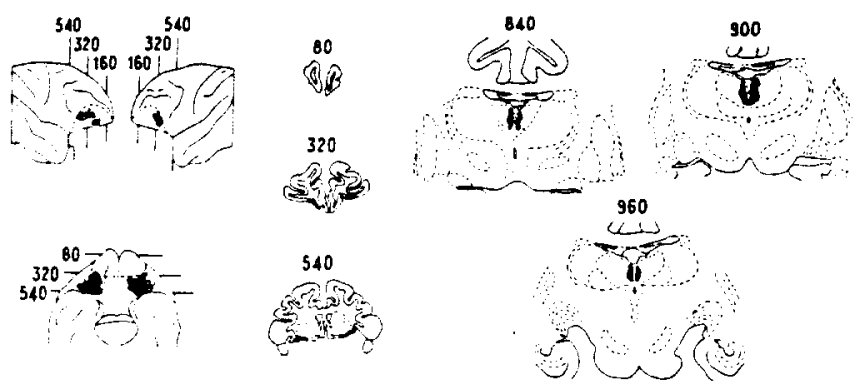

FIG. 1D

$2.12 ; d f=45 / 66 ; p<0.01$ ). Comparisons of individual groups by the Newman-Keuls test [17] revealed that both the OF and PMOF groups made significantly more responses in extinction than did either the $N$ or DLF groups ( $p<0.05$, for all comparisons), while the latter two groups were not different from each other. The differences between the AOF and LOF groups, on the one hand, and the OF and PMOF groups on the other, approached significance $(p<0.10)$. The DLF, $A O F$ and LOF groups were not reliably different from the $\mathrm{N}$ group.

The results of this experiment confirm prior findings [4] concerning the effects of OF and DLF lesions on operant responding. In this study, as in the previous one, OF lesions did not alter responding reinforced on a VI schedule, but unlike DLF lesions, did produce heightened responding in extinction. Moreover, the OF monkeys, like those in the previous experiment, developed abnormally high rates of responding over several extinction sessions.
With regard to the partial OF lesions, the PMOF lesions were the only ones which produced a significant enhancement of extinction responses. Furthermore, the PMOF monkeys, like the OF monkeys, showed increasing response rates over successive extinction sessions. This finding, then, supports the prediction stated previously: One aspect of the perseverative syndrome seen following OF removal-increased extinction responses-is selectively associated with PMOF removal.

\section{EXPERIMENT II}

In the prior experiment, as was anticipated, the DLF monkeys did not show abnormally enhanced extinction responses. Previous findings suggest that while DLF removal does not affect suppression of a variety of other response tendencies as severely as does OF removal, DLF removal is 


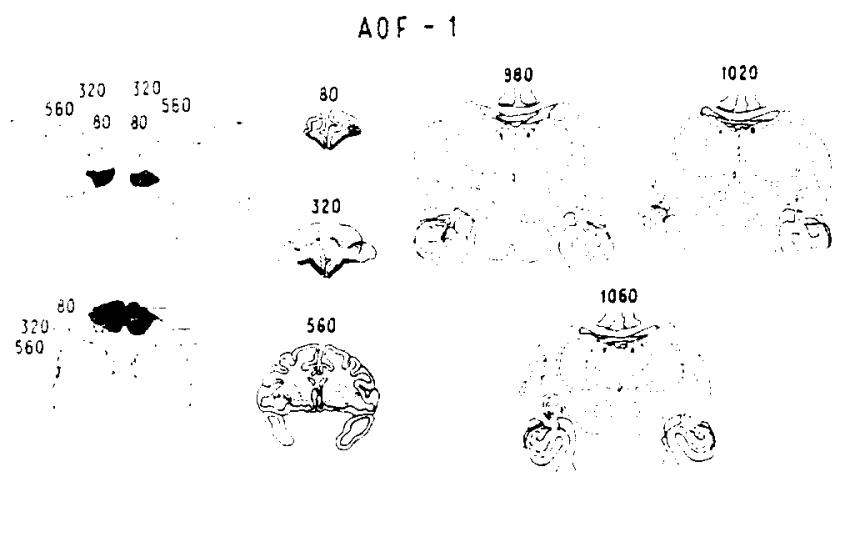

$A O F-2$
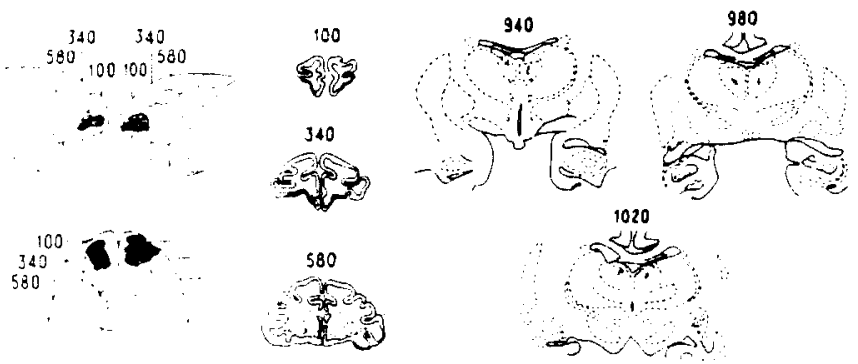

$A O F-3$
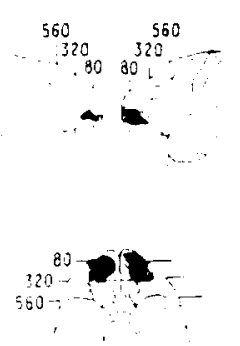
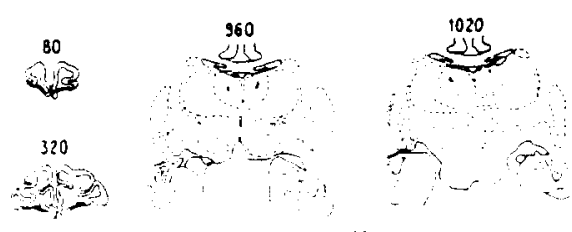

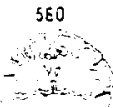

1060

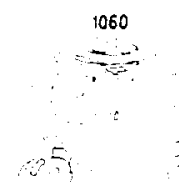

FIG. IE

FIG. 1. Reconstructions of the different frontal lesions together with representative cross-sections through the lesions and portions of the thalamus containing retrograde degeneration.

DLF lesions (A); OF lesions (B); LOF lesions (C); PMOF lesions (D); AOF lesions (E).

selectively associated with deficits in spatial tasks [10]. The present experiment was undertaken in order to confirm this i iew.

\section{METHOD}

\section{Subjects and Apparatus}

Subjects were the same ones used in the previous experiment. The animals were tested in a standard Wisconsin General Test Apparatus (WGTA) described in detail in a prior report [3]. The WGTA contained a stimulus-tray with two foodwells, 13 in. from center to center. Gray, poster-

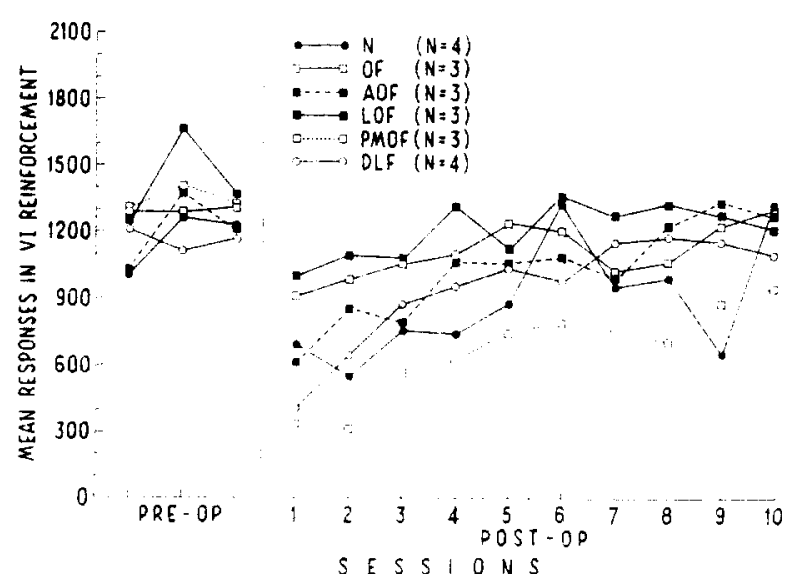

FIG. 2. Mean response rates of the 6 groups in the last three preoperative and the 10 postoperative VI-reinforcement sessions.

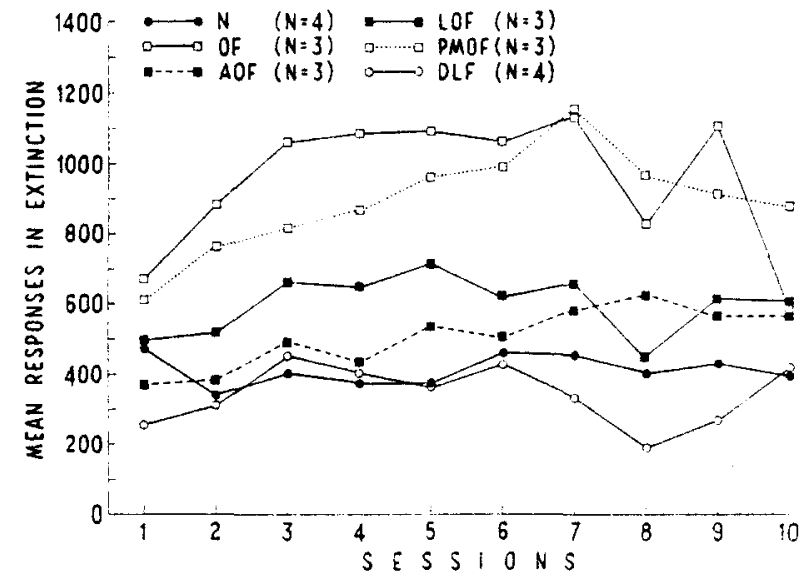

FIG. 3. Mean response rates of the 6 groups in the 10 postoperative extinction sessions.

board plaques, 3 in. square, served as stimuli. Testing was conducted in a darkened room, and outside noises were masked by fans.

\section{Procedure}

Approximately 3 weeks following the completion of extinction testing, the monkeys were trained to displace plaques in order to obtain one-half a peanut. They were then given 30 trials on which displacement of either the left or the right plaque was rewarded. If an animal responded on one side more than 3 times in succession, only the opposite foodwell was baited on subsequent trials until the animal responded once on that side. This procedure was employed in order to prevent the development of position preferences that would affect spatial discrimination learning.

On the day following the completion of plaque-displacement training, the monkeys began spatial discrimination training, in which they were rewarded only for displacing the plaque on the right side. Intertrial intervals were approximately $5 \mathrm{sec}$ in duration, and 30 trials were administered daily until the monkeys responded correctly on 27 trials in a single session. In the 
following session, the animals were rewarded only for displacing the left plaque. Discrimination reversal testing was conducted in the same manner as was original discrimination training. Upon reattainment of the criterion of 27 correct responses in 30 trials, the spatial discrimination problem was reversed again. In all, the animals were administered 5 reversals, in each of which the procedure was identical to that employed in initial training.

\section{RESULTS AND DISCUSSION}

Figure 4 shows the mean errors of each of the groups in spatial discrimination training and in each of the 5 reversals. As seen in the figure, the different frontal lesions did not affect performance on the reversal problems equally, and the lesion effects were different from those found in the previous experiment. Thus the AOF group was not impaired in any of the stages of testing, while the PMOF group performed on the average only slightly worse than did the $\mathrm{N}$ group on reversals $2-5$. On the other hand, the DLF and LOF groups committed many more errors than did the N, PMOF or AOF groups on the first two reversals. Although their performance improved following the second reversal, the DLF and LOF groups still made 4-5 times as many errors as did the N, PMOF or AOF animals in the last three reversals. The OF group showed a different pattern of impairment than did the DLF and LOF groups: The OF animals were obviously not impaired in the first reversal, but, unlike the $\mathrm{N}$ animals, they failed to improve their performance; rather, they showed a tendency to make somewhat more errors in subsequent reversals. An analysis of variance of the groups' errors in learning and in each of the reversals indicates a significant lesion effect $(F=4.20 ; d f=5 / 14 ; p<0.05)$ and a lesion by stages interaction which approached significance $(F=1.68$; $d f=25 / 70 ; p<0.10$ ). Moreover, a comparison of individual groups' performance in all stages of testing by the NewmanKeuls procedure reveals that both the LOF and DLF groups committed significantly more errors than did either the $N$, PMOF or AOF groups ( $p<0.05$ for all comparisons), while the errors of the latter three groups were not reliably different from each other. The difference between the mean errors of the $\mathrm{N}$ and $\mathrm{OF}$ groups narrowly missed statistical significance.

Finally, the analyses summarized in Table 1 indicate that the groups that were impaired in the spatial reversal tests also

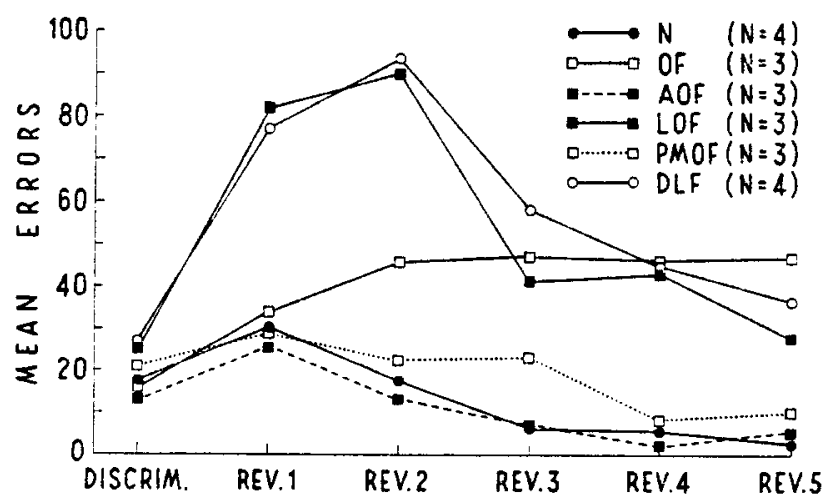

FIG. 4. Mean errors of the 6 groups in spatial discrimination learning and in the 5 reversals. made abnormally large numbers of perseverative errors and abnormally long perseverative runs. Thus all of the monkeys in the OF, LOF and DLF groups showed higher scores on both of these measures of perseveration than did any of the $\mathrm{N}$, PMOF or AOF monkeys.

TABLE 1.

Average Perseverative Errors (PE) and PERSEVERATIVE ERRor RUNS ${ }^{1}$ (PE RUNS) on all Spatial Reversals

\begin{tabular}{lrrrrrr}
\hline & \multicolumn{6}{c}{ Groups } \\
& $\mathrm{N}$ & OF & PMOF & LOF & AOF & DLF \\
\hline PE & 7.6 & $36.2^{2}$ & 11.8 & $45.6^{2}$ & 8.0 & $49.2^{2}$ \\
PE Runs 2.6 & $5.1^{2}$ & 3.2 & 10.0 & 2.7 & $5.2^{2}$ \\
\hline
\end{tabular}

1 Perseverative error runs defined as two or more consecutive errors.

${ }^{2}$ Individual scores not overlapping with those of $\mathrm{N}$ animals.

As expected on the basis of previous findings, the DLF monkeys were severely impaired in spatial reversal performance compared with the unoperated controls. Moreover, these animals also showed abnormal perseveration of the previously correct response on reversal tests. While the monkeys with OF ablation also showed abnormally heightened perseverative tendencies, they were not as severely impaired as were the DLF monkeys on the first two reversals. This dissociation of impairments produced by DLF and OF lesions, together with the prior findings that DLF lesions do not alter extinction, supports the view that DLF cortex is selectively associated with spatial factors [10].

On the other hand, the OF monkeys' unimpaired performance on the initial reversal test suggests that these animals were capable of normally utilizing spatial cues. Since these animals, unlike the unoperated controls, failed to improve their performance over the last four reversals, it would appear that they were impaired in utilizing the information provided by previous reversals.

The finding that the LOF animals were as severely impaired as were the DLF animals in reversals 1 and 2 appears puzzling, since those with total OF removal did not show this pattern of impairment. However, these apparently conflicting findings are clarified by the histological analyses which, as mentioned previously, indicate that the LOF lesions, unlike the other partial OF removals, involved moderate damage to DLF cortex (see Fig. 1), including the banks and depths of sulcus principalis. In fact, there is a close relationship between DLF damage and spatial reversal performance in the LOF animals. Of the three LOF monkeys, the one which sustained the most damage to the dorsal convexity and to sulcus principalis (LOF-1) was the most severely impaired in reversal tests, while the one which sustained the least damage to these structures (LOF-3) was the least impaired. On the other hand, the finding that total OF removal, but not AOF or PMOF removal, severely impairs spatial reversal performance suggests that LOF ablation itself may contribute to these reversal deficits. 


\section{EXPERIMENT III}

The results of the first experiment indicate that the abnormal perseveration in extinction seen following total OF ablation may be attributed to PMOF removal. The purpose of this experiment was to determine whether PMOF removal is also selectively associated with deficits in another situation where OF removal produces perseverative impairments-object discrimination reversal.

\section{METHOD}

\section{Subjects and Apparatus}

The same monkeys tested in the prior experiments served as subjects in this experiment. They were trained and tested in the WGTA used in Experiment 2.

\section{Procedure}

Approximately 10 days following the completion of spatial reversal testing, the monkeys were trained in the WGTA to discriminate between two objects, a toy metal pistol and a curved copper pipe, which were presented simultaneously over the two foodwells of the stimulus tray. Displacement of the gun was rewarded with one-half a peanut, while displacement of the pipe was unrewarded. Thirty trials were administered daily and the spatial position of the stimuli was determined by a Gellerman series [6]. Intertrial intervals were approximately $5 \mathrm{sec}$ in duration. Daily discrimination training sessions were continued until the animals performed at least 29 correct responses in two consecutive sessions. On the day following the completion of discrimination training, the animals received discrimination reversal testing, in which only the previously unrewarded object (the pipe) was now rewarded. In all other respects, the procedure was identical to that employed in discrimination training. Following the attainment of the criterion, the significance of the stimuli was again reversed 4 more times in succession, so that a total of 5 object reversals were administered.

\section{RESULTS}

Figure 5 shows the mean errors of each group in discrimination learning and in each of the 5 reversals. As in prior spatial reversal testing, the LOF groun made many more errors than

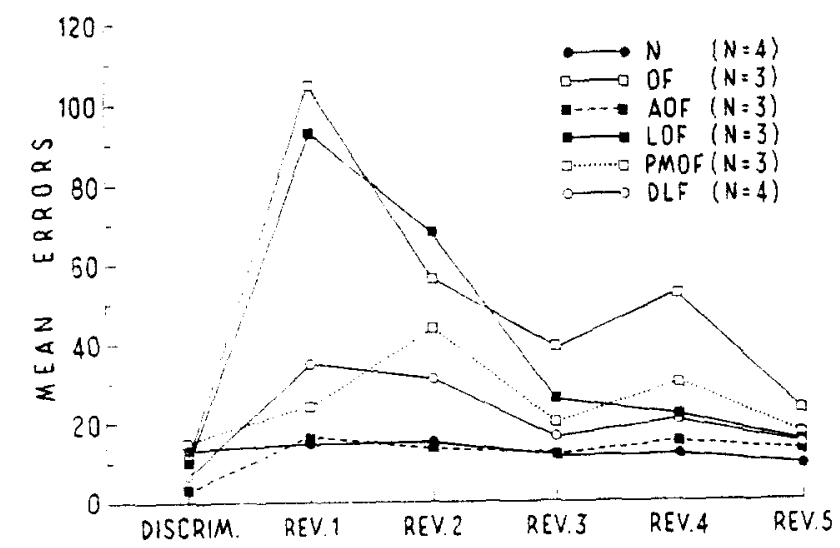

FIG. 5. Mean errors of the 6 groups in object discrimination learning and in the 5 reversals. did the $\mathrm{N}$ group on the first 2 reversals, but subsequently improved their performance. However, in comparison with their performance in spatial reversal testing, it is apparent that the OF and DLF groups reversed their relative performance: Here, the OF group made approximately as many errors as did the LOF group, whereas the DLF group was much less impaired. Moreover, the PMOF group committed somewhat more errors than did the $\mathrm{N}$ group, whereas the AOF group was obviously unimpaired.

Analysis of variance of the findings presented in Fig. 5 disclosed that the lesion effect was highly significant $(\mathrm{F}=$ $7.70 ; d f=5 ; 14 ; p<0.01)$, and the lesion by stage interaction was also significant $(F=1.64 ; d f=25 / 70 ; p<0.05)$. The lesion effect was further analyzed by comparing individual group's errors averaged over all stages of the experiment by the Newman-Keuls procedure. These comparisons revealed that both the OF and LOF groups made significantly more errors than did the $N$ group $(p<0.01$ and $p<0.05$, respectively). The $O F$ group also performed significantly worse than did the DLF or AOF groups $(p<0.05$ for both comparisons), while the LOF group made reliably more errors than did the AOF group $(p<0.05)$. On the other hand, the mean errors of the DLF, PMOF and AOF groups did not differ from those of the $\mathrm{N}$ group.

TABLE 2.

Average Perseverative ERrors (PE) aNi) Perseverative Error Runs' (PE Runs) on ALl. OBject ReVErsals

\begin{tabular}{lrrrrrr}
\hline & \multicolumn{6}{c}{ Groups } \\
& \multirow{2}{*}{$\mathrm{N}$} & OF & PMOF & LOF & AOF & DLF \\
\hline PE & 6.3 & $33.1^{*}$ & 19.3 & $28.9^{2}$ & 7.0 & $17.3^{2}$ \\
PE Runs 3.2 & $9.7^{2}$ & 5.7 & $9.3^{2}$ & 3.3 & $8.9^{2}$ \\
\hline
\end{tabular}

\footnotetext{
1 Perseverative error runs defined as two or more consecutive errors.

"Individual scores not overlapping with those of $\mathrm{N}$ animals.
}

The results presented in Table 2 indicate that both the OF and LOF animals made more perseverative errors and longer perseverative error runs than did the $\mathrm{N}$ animals. Moreover, although the DLF monkeys did not reliably differ from the unoperated monkeys in the analyses described previously, they were impaired on these measures of perseveration.

\section{GENERAL DISCLSSION}

The finding that the animals with OF lesions performed more poorly than did those with DLF lesions in object discrimination reversal is consistent with previous findings [10]. This result, together with the more severe spatial reversal impairment found in the DLF than in the OF monkeys. adds further support to the view that DLF cortex is selectively related to performance on spatial tasks.

While the PMOF monkeys were the only ones with partial lesions that were impaired in extinction, they were not reliably impaired in object reversal testing. Rather, only the LOF animals showed the marked retardation in object reversal 
testing exhibited by the animals with total OF removal. It is possible that the LOF monkeys' impairment in object reversal, like their severe impairment in spatial reversal, was due to partial destruction of DLF cortex. However, there was no correlation between errors in object reversal testing and degree of damage to DLF cortex in the LOF monkeys. This finding suggests that the LOF deficit in object reversal is not simply due to DLF damage alone. However, there is also evidence that the portion of DLF cortex adjacent to OF cortex, on the dorsal convexity, may be critically involved in object reversal performance. Iversen and Mishkin [7] reported that monkeys with removal of LOF cortex plus the adjoining cortex on the dorsal convexity were more severely impaired in object reversal testing than were monkeys with medial orbital lesions, which included PMOF cortex. Thus, it is likely that the ventral portion of DLF cortex, which was partially damaged in the LOF animals, together with LOF cortex itself does contribute to object reversal performance.

While the lateral portion of OF cortex appears to be specifically related to object reversal performance, its removal did not impair extinction performance, whereas PMOF removal enhanced responses in extinction. This double dissociation of OF lesions and performance deficits indicates that the "perseverative syndrome" produced by total OF removal can be fractionated and provides evidence for differential localization of functions in OF cortex. However, it is not clear from these findings what the nature of the impairments are underlying the deficits produced by PMOF and LOF ablation. Since the object reversal situation involved a two-choice method of testing, whereas the extinction test involved responding vs. not responding, it is possible that the response requirements of the task might be relevant in obtaining deficits following LOF or PMOF ablation. However, it is unlikely that this factor was crucial to the dissociation of deficits found here. For, monkeys with LOF lesions are more impaired than those with lesions involving PMOF cortex in the retention of an auditory differentiation task, involving the go-no go method of testing [7]. Of course, there are many other ways in which the object reversal and extinction tasks differ from each other, and only future experiments can decide which of these are critical to the dissociation of LOF and PMOF deficits. One factor which might be relevant in specifying the role of PMOF cortex in suppressing response tendencies is the close association between this region and limbic structures. In fact, anatomically PMOF cortex is more properly considered transitional cortex, like other limbic regions, rather than neocortex [13]. It is possible, then, that PMOF cortex, like other limbic structures, contributes to motivational processes which might be involved in behavioral suppression.

\section{REFERENCES}

1. von Bonin, G. and P. Bailey. The Neocortex of Macaca Mulatra. Univ. Illinois Press, Urbana, Ill., 1947.

2. Brutkowski, S., M. Mishkin and H. E. Rosvold. Positive and inhibitory motor CRs in monkeys after ablation of orbital or dorsolateral surface of the frontal cortex. In: Second Symposium on Peripheral and Central Mechanisms of Motor Functions, edited by E. Gutman. Liblice, Czechoslovakia:Czechoslovakian Academy of Sciences, 1963.

3. Butter, C. M. The effect of discrimination training on pattern equivalence in monkeys with inferotemporal and lateral striate lesions. Neuropsychologia $6: 27-40,1968$.

4. Butter, C. M., M. Mishkin and H. E. Rosvold. Conditioning and extinction of a food-rewarded response after selective ablations of frontal cortex in rhesus monkeys. Expl Neurol. 7: 65-75, 1963.

5. Dorff, J. E. The effect of combined subcortical lesions in monkeys on delayed alternation, visual discrimination and the extinction of a bar pressing response. Unpublished Doctoral Dissertation, Catholic University, 1964.

6. Gellerman, L. W. Chance orders of alternating stimuli in visual discrimination experiments. $J$. genet. Psychol. 42: 207-208, 1933.

7. Iversen, S., M. Mishkin and H. E. Rosvold. Perseverative interference in monkeys following selective lesions of the inferior frontal convexity. Presented at Eastern Psychological Association, New York, April, 1966.

8. Kaada, B. R. Cingulate, posterior orbital, anterior insular and temporal pole cortex. In: Handbook of Physiol., Vol. II, edited by J. Field. Washington, D.C.: Amer. Physiol. Soc., 1960.

9. Mahut, $\mathrm{H}$. and J. P. Cordeau. Spatial reversal deficit in monkeys with amygdalohippocampal ablations. Expl Neurol. 7: 426-434, 1963.

10. Mishkin, M. Perseveration of central sets after frontal lesions in monkeys. In: The Frontal Granular Cortex and Behavior, edited by J. M. Warren and K. Akert. New York: McGrawHill, 1964.

11. Nauta, W. J. H. Neural associations of the amygdaloid complex in the monkey. Brain 85: 505-520, 1962.

12. Pribram, K. H., K. L. Chow and J. Semmes. Limit and organization of the cortical projection from the medial thalamic nucleus in monkey. J. comp. Neurol. 98: 433-448, 1953.

13. Pribram, K. H. and L. Kruger. Functions of the "Olfactory Brain", Annls N.Y. Acad. Sci. 58: 109-138, 1954.

14. Rosvold, H. E. and M. Mishkin. Non-sensory effects of frontal lesions on discrimination learning and performance. In: Brain Mechanisms and Learning, edited by J. F. Delafresnaye. Oxford: Blackwell Scientific Publications, 1961.

15. Walker, A. E. A cytoarchitectural study of the prefrontal area of the macaque monkey, J. comp. Neurol. 73: 59-86, 1940.

16. Weiskrantz, L. Behavioral changes associated with ablation of the amygdaloid complex in monkeys. J. comp. physiol. Psychol. 49: 381-391, 1956.

17. Winer, B. J. Statistical Principles in Experimental Design. New York: McGraw-Hill, 1962. 